\title{
Status of Dynamical Coupled-Channel analysis by Collaboration@EBAC
}

\author{
T.-S. H. Lee \\ Physics Division, Argonne National Laboratory, Argonne, Illinois 60439, USA
}

\begin{abstract}
The development and results of the Dynamical Coupled-Channels analysis by a collaboration at the Excited Baryon Analysis Center (EBAC) are reported.
\end{abstract}

Keywords: Nucleon resonance analysis

PACS: 14.20.Gk, 13.75.Gx, 13.60.Le

\section{INTRODUCTION}

In this contribution, we report on the development and results from a dynamical coupledchannel (DCC) analysis by a collaboration associated with the Excited Baryon Analysis Center (EBAC) at Jefferson Lab during the period January, 2006 - March, 2012. This project has three components : (1) perform a dynamical coupled-channels analysis on the world data on meson production reactions from the nucleon to determine the meson-baryon partial-wave amplitudes, (2) extract the $N^{*}$ parameters from the determined partial-wave amplitudes, and (3) investigate the interpretations of the extracted $N^{*}$ properties in terms of the available hadron models and Lattice QCD.

\section{MODEL}

The DCC analysis by the collaboration@EBAC is based on an extension [1] of the dynamical model for the $\Delta(1232)$ resonance developed [2] by an Argonne National Laboratory-Osaka University (ANL-Osaka) collaboration. Within the extended ANLOsaka formulation [1], the reaction amplitudes $T_{\alpha, \beta}\left(p, p^{\prime} ; E\right)$ in each partial-wave are calculated from the following coupled-channels integral equations,

$$
\begin{aligned}
T_{\alpha, \beta}\left(p, p^{\prime} ; E\right) & =V_{\alpha, \beta}\left(p, p^{\prime}\right)+\sum_{\gamma} \int_{0}^{\infty} q^{2} d q V_{\alpha, \gamma}(p, q) G_{\gamma}(q, E) T_{\gamma, \beta}\left(q, p^{\prime}, E\right) \\
V_{\alpha, \beta} & =v_{\alpha, \beta}+\sum_{N^{*}} \frac{\Gamma_{N^{*}, \alpha}^{\dagger} \Gamma_{N^{*}, \beta}}{E-M^{*}}
\end{aligned}
$$

where $\alpha, \beta, \gamma=\gamma N, \pi N, \eta N, K Y, \omega N$, and $\pi \pi N$ which has $\pi \Delta, \rho N, \sigma N$ resonant components, $v_{\alpha, \beta}$ are meson-exchange interactions deduced from phenomenological Lagrangian, $\Gamma_{N^{*}, \beta}$ describes the excitation of the nucleon to a bare $N^{*}$ state with a mass $M^{*}$, and $G_{\gamma}(q, E)$ is a meson-baryon propagator. The DCC model, defined by Eqs. (1) and (2), satisfies two- and three-body unitarity conditions. 
In order to determine the parameters associated with the strong-interaction parts of $V_{\alpha, \beta}$ in Eq. (2), the considered DCC model was first applied to fit the $\pi N$ elastic scattering up to invariant mass $W=2 \mathrm{GeV}$. For simplicity, $K Y$ and $\omega N$ channels were not included during the development stage in 2006-2010. The electromagnetic parts of $V_{\alpha, \beta}$ were then determined by fitting the data of $\gamma p \rightarrow \pi^{0} p, \pi^{+} n$ and $p\left(e, e^{\prime} \pi^{0,+}\right) N$.

The resulting 6-channel model was then tested by comparing the predicted $\pi N, \gamma N \rightarrow$ $\pi \pi N$ production cross sections with the data. In parallel to analyzing the data, a procedure to analytically continue Eqs. (1) and (2) to the complex energy plane was developed to extract the positions and residues of nucleon resonances.

In the following, we present a sample of results from these efforts.

\section{Results for single pion production reactions}

In fitting the $\pi N$ elastic scattering, we found that one or two bare $N^{*}$ states were needed in each partial wave. The coupling strengths of the $N^{*} \rightarrow M B$ vertex interactions $\Gamma_{N^{*}, M B}$ with $M B=\pi N, \eta N, \pi \Delta, \rho N, \sigma N$ were then determined in the $\chi^{2}$-fits to the data. Our results were given in Ref. [3].

Our next step was to determine the bare $\gamma N \rightarrow N^{*}$ interaction $\Gamma_{N^{*}, \gamma N}$ by fitting the $\gamma p \rightarrow \pi^{0} p$ and $\gamma p \rightarrow \pi^{+} n$ data. We found [4] that we were able to fit the data only up to invariant mass $W=1.6 \mathrm{GeV}$, mainly because we did not adjust any parameter which was already fixed in the fits to $\pi N$ elastic scattering. Some of our results for total cross sections $(\sigma)$, differential cross sections $(d \sigma / d \Omega)$, and photon asymmetry $(\Sigma)$ are shown in Fig. 1.

The $Q^{2}$-dependence of the $\Gamma_{N^{*}, \gamma N}$ vertex functions were then determined [5] by fitting the $p\left(e, e^{\prime} \pi^{0}\right) p$ and $p\left(e, e^{\prime} \pi^{+}\right) n$ data up to $W=1.6 \mathrm{GeV}$ and $Q^{2}=1.5(\mathrm{GeV} / \mathrm{c})^{2}$.

\section{Results for two-pions production reactions}

The model constructed from fitting the data on single pion production reactions was then tested by examining the extent to which the $\pi N \rightarrow \pi \pi N$ and $\gamma N \rightarrow \pi \pi N$ data can be described. It was found $[6,7]$ that the predicted total cross sections are in excellent agreement with the data in the near threshold region $W \leq 1.4 \mathrm{GeV}$. In the higher $W$ region, the predicted $\pi N \rightarrow \pi \pi N$ cross sections can describe to a very large extent the available data, as shown in Fig. 2. Here the important role of the coupled-channel effects were also demonstrated. However, the predicted $\gamma p \rightarrow \pi^{+} \pi^{-} p, \pi^{0} \pi^{0} p$ cross sections were a factor of about 2 larger than the data while the shapes of two-particles invariant mass distributions could be described very well.

\section{Resonance Extractions}

We follow earlier work, as reviewed and explained in Refs. [8, 9], to define that the resonances are the eigenstates of the Hamiltonian with only outgoing waves of their 

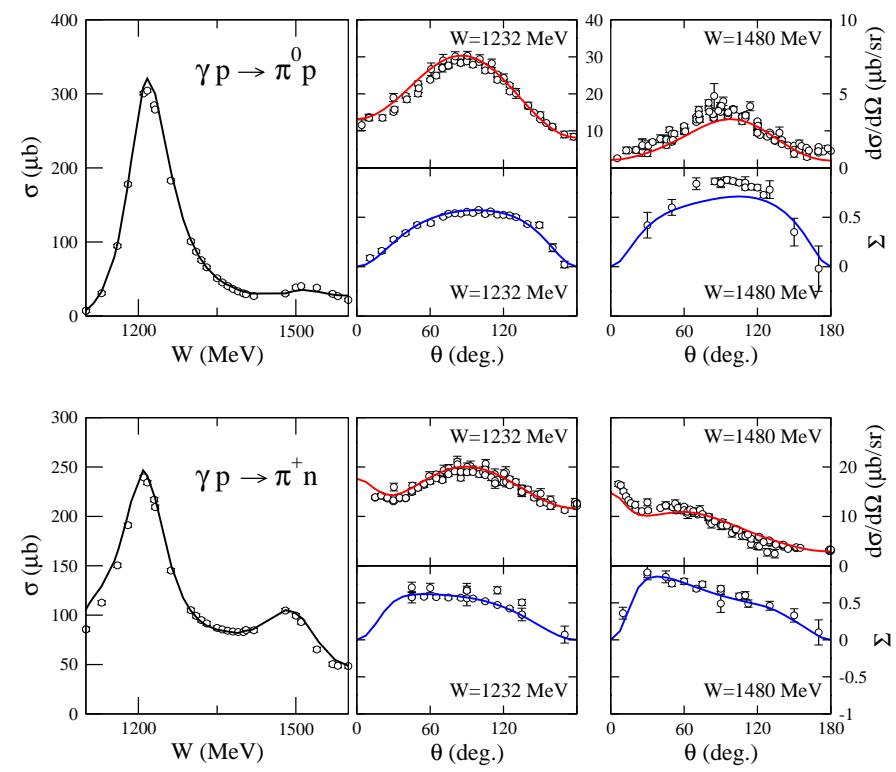

FIGURE 1. The DCC results [4] of total cross sections $(\sigma)$, differential cross sections $(d \sigma / d \Omega)$, and photon asymmetry ( $\Sigma$ ) of $\gamma p \rightarrow \pi^{0} p$ (upper parts), $\gamma p \rightarrow \pi^{+} n$ (lower parts).
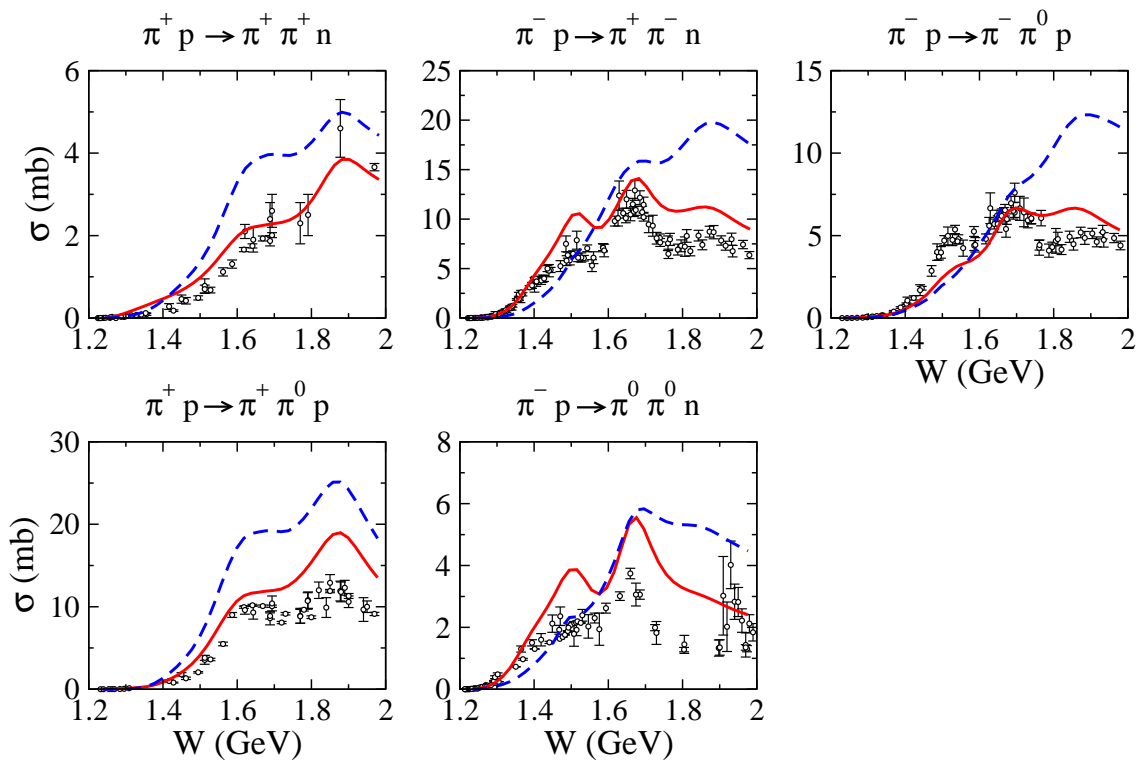

FIGURE 2. The predicted [6] total cross sections of the $\pi N \rightarrow \pi \pi N$ are compared with the data. The dashed-curves are obtained when the coupled-channel effects are turned off within the DCC model of Ref.[1].

decay channels. One can then show that the nucleon resonance positions are the poles $M_{R}$ of meson-baryon scattering amplitudes calculated from Eqs. (1) and (2) on the unphysical sheets of complex- $E$ Riemann surface. The coupling of meson-baryon states with the resonances can be determined by the residues $R_{N^{*}, M B}$ at the pole positions. Our 


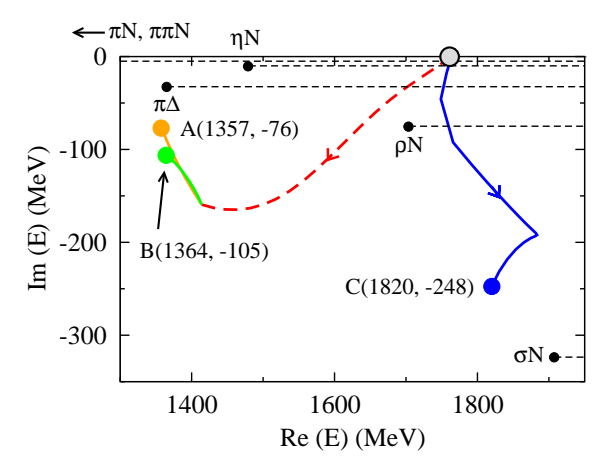

FIGURE 3. The trajectories of the evolution of three nucleon resonances in $P_{11}$ from the same bare $N^{*}$ state. The results are from Ref. [10].

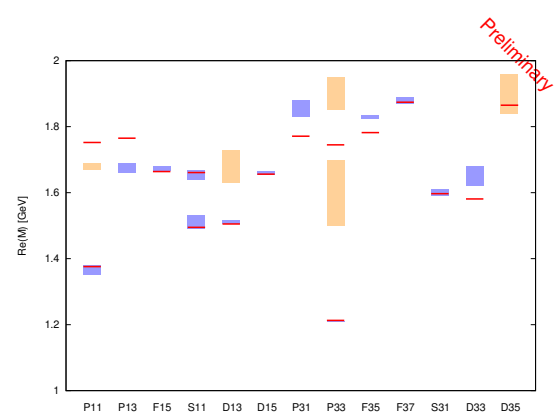

FIGURE 4. Preliminary results (red bars) of the determined $N^{*}$ spectrum are compared with 4-star (blue bands) and 3-star (brown bands) states listed by the Particle Data Group.

procedures for determining $M_{R}$ and $R_{N^{*}, M B}$ and the results were presented in Refs. [8, 9, $10,11]$.

With our analytic continuation method [8,9], we were able to analyze the dynamical origins of the nucleon resonances extracted from the considered DCC model of Ref.[1]. This was done by examining how the resonance positions move as the coupled-channels effects are gradually turned off. As illustrated in Fig. 3 for the $P_{11}$ states, this exercise revealed that the two poles in the Roper region and the next higher pole are associated with the same bare state.

\section{PROSPECT}

During the developing stage of DCC analysis by Collaboration@EBAC in 2006-2010, the DCC model parameters were determined by analyzing separately the following data: $\pi N \rightarrow \pi N$ [3], $\gamma N \rightarrow \pi N$ [4], $N\left(e, e^{\prime} \pi\right) N$ [5], $\pi N \rightarrow \pi \pi N$ [6], and $\gamma N \rightarrow \pi \pi N$ [7]. The very extensive data on $K \Lambda$ and $K \Sigma$ production were not included in the analysis. To have a high precision extraction of nucleon resonances, it is necessary to perform a combined simultaneous coupled-channels analysis of all meson production reactions.

We started an 8-channels combined analysis of world data of $\pi N, \gamma N \rightarrow$ 
$\pi N, \eta N, K \Lambda, K \Sigma$ since the summer of 2010 . Only the preliminary results had been obtained by March 31, 2012 when the Collaboration@EBAC was dissolved. In Fig.4, we show the determined excited nucleon spectrum from this combined analysis. An ANL-Osaka collaboration has since taken over this task and we expect to complete the analysis by the end of 2012. The ANL-Osaka analysis will then proceed to extracting $\gamma N \rightarrow N^{*}$ form factors up to sufficiently high $Q^{2}$ from the new JLab data on meson electroproduction data. In addition, we will explore the interpretations of the extracted resonance parameters in terms of available hadron models, such as the Dyson-Schwinger-Equation model and constituent quark model, and Lattice QCD. This last step is needed to complete the DCC project with conclusive results, as discussed in Refs.[1, 2, 10, 12].

\section{ACKNOWLEDGMENTS}

The author thanks B. Juliá-Díaz, H. Kamano, A. Matsuyama, S. X. Nakamura, T. Sato, and N. Suzuki for their collaborations at EBAC, and would also like to thank A. W. Thomas for his strong support and his many constructive discussions. This work is supported by the U.S. Department of Energy, Office of Nuclear Physics Division, under Contract No. DE-AC02-06CH11357, and Contract No. DE-AC05-06OR23177 under which Jefferson Science Associates operates the Jefferson Lab. This research used resources of the National Energy Research Scientific Computing Center, which is supported by the Office of Science of the U.S. Department of Energy under Contract No. DE-AC02-05CH11231, resources provided on "Fusion," a 320-node computing cluster operated by the Laboratory Computing Resource Center at Argonne National Laboratory, and resources of Barcelona Sucpercomputing Center (BSC/CNS).

\section{REFERENCES}

1. A. Matsuyama, T. Sato, and T.-S. H. Lee, Phys. Rept. 439, 193-253 (2007).

2. T. Sato and T.-S. H. Lee, Phys. Rev. C54,2660 (1996)

3. B. Juliá-Díaz, T.-S. H. Lee, A. Matsuyama, and T. Sato, Phys. Rev. C 76, 065201 (2007).

4. B. Juliá-Díaz, T.-S. H. Lee, A. Matsuyama, T. Sato, and L. C. Smith, Phys. Rev. C 77, 045205 (2008).

5. B. Juliá-Díaz, H. Kamano, T.-S. H. Lee, A. Matsuyama, T. Sato, and N. Suzuki, Phys. Rev. C 80, 025207 (2009).

6. H. Kamano, B. Juliá-Díaz, T.-S. H. Lee, A. Matsuyama, and T. Sato, Phys. Rev. C 79, 025206 (2009).

7. H. Kamano, B. Juliá-Díaz, T.-S. H. Lee, A. Matsuyama, and T. Sato, Phys. Rev. C 80, 065203 (2009).

8. N. Suzuki, T. Sato, and T.-S. H. Lee, Phys. Rev. C 79, 025205 (2009).

9. N. Suzuki, T. Sato, and T.-S. H. Lee, Phys. Rev. C 82, 045206 (2010).

10. N. Suzuki, B. Juliá-Díaz, H. Kamano, T.-S. H. Lee, A. Matsuyama, and T. Sato, Phys. Rev. Lett. 104, $042302(2010)$.

11. H. Kamano, S. X. Nakamura, T.-S. H. Lee, and T. Sato, Phys. Rev. C 81, 065207 (2010).

12. D. J. Wilson, I. C. CloÃnt, L. Chang and C. D. Roberts Phys. Rev. Phys. Rev. C 85. 025205 (2012) 